\title{
Blood Utilization After Primary Total Joint Arthroplasty in a Large Hospital Network
}

\author{
Antonia F. Chen, MD, MBA • Brian A. Klatt, MD • Mark H. Yazer, MD • Jonathan H. Waters, MD
}

Received: 1 October 2012/Accepted: 4 January 2013/Published online: 21 June 2013

(C) The Author(s) 2013. This article is published with open access at Springerlink.com

\begin{abstract}
Background: Since a study in orthopedic hip fracture patients demonstrated that a liberal hemoglobin $(\mathrm{Hb})$ threshold does not improve patient morbidity and mortality relative to a restrictive $\mathrm{Hb}$ threshold, the standard of care in total joint arthroplasty (TJA) should be examined to understand the variability of red blood cell (RBC) transfusion following TJA. Questions/purposes: The study aimed to answer the following questions: (1) What is the blood utilization rate after primary TJA for individual surgeons within a large hospital network? (2) What is the comparison of hospital charges, length of stay (LOS), and discharge locations among TJA patients who were and were not transfused? Methods: A retrospective study was conducted on 3,750 primary total knee arthroplasties (TKAs) and 2,070 primary total hip arthroplasties (THAs), and data was retrospectively collected over a 15 -month period on the number
\end{abstract}

This work was performed at the University of Pittsburgh Medical Center.
Level of evidence: Therapeutic study, level III (retrospective study).

Electronic supplementary material The online version of this article (doi:10.1007/s11420-013-9327-y) contains supplementary material, which is available to authorized users.

\author{
A. F. Chen, MD, MBA • B. A. Klatt, MD $(\bowtie)$ \\ Department of Orthopaedic Surgery, \\ University of Pittsburgh Medical Center, \\ 5230 Centre Avenue, Suite 415, Pittsburgh, PA 15232, USA \\ e-mail:klattba@upmc.edu \\ M. H. Yazer, MD \\ Department of Pathology, \\ University of Pittsburgh Medical Center, \\ 5230 Centre Avenue, Suite 415, Pittsburgh, PA 15232, USA
}

M. H. Yazer, MD

The Institute for Transfusion Medicine,

3636 Blvd. of the Allies, Pittsburgh, PA 15213, USA

\section{J. H. Waters, MD}

Department of Anesthesiology,

University of Pittsburgh Medical Center,

300 Halket Street, Suite 3510, Pittsburgh, PA 15213, USA of RBCs transfused per patient, along with demographic and cost details. The number of patients who received at least 1 RBC unit and the number of RBCs transfused per patient was calculated and stratified by surgeon. Results: In the postoperative period, $19.3 \%$ TKA patients and $38.5 \%$ THA patients received a RBC transfusion. Transfusion rates following TJA varied widely between surgeons (TKA 4.8$63.8 \%$, THA $4.3-86.8 \%$ ). Transfused TKA patients received an average of $1.65 \pm 0.03 \mathrm{RBCs}$, and THA patients received an average of $1.97 \pm 0.14$ RBCs. LOS and hospital charges for blood transfusion patients were higher than nontransfused patients. Conclusion: Blood utilization after primary TJA varies greatly among surgeons, suggesting that resources may be misallocated. These findings highlight the need to standardize RBC transfusion practice following TJA.

Keywords total joint arthroplasty.blood utilization. transfusion rate $\cdot$ blood management $\cdot$ red blood cell (RBC) transfusion · intervention

\section{Introduction}

The threshold for red blood cell (RBC) transfusion in the postoperative surgical period has evolved over the years. In 1942 , it was believed that it was ideal to maintain a hemoglobin $(\mathrm{Hb})$ and hematocrit (Hct) over $10.0 \mathrm{~g} / \mathrm{dL}$ and $30.0 \mathrm{~g} / \mathrm{dL}$, respectively, in high-risk surgical patients [1]. The need for liberal blood transfusion $(\mathrm{Hb}>10.0 \mathrm{~g} / \mathrm{dL})$ was questioned when a randomized, controlled trial comparing a liberal to a restrictive $\mathrm{RBC}$ transfusion policy (transfuse if $\mathrm{Hb}<7.0 \mathrm{~g} / \mathrm{dL}$ ) in hemodynamically stable intensive care unit (ICU) patients demonstrated that there was no difference in 30-day mortality or in cardiac events regardless of the $\mathrm{Hb}$ threshold employed (Transfusion Requirements in Critical Care study, TRICC) [10]. Thus began a trend towards lower RBC transfusion thresholds in critically ill and general medicine patients.

A large, multicenter, randomized study evaluating transfusion triggers in postoperative hip fracture patients with cardiovascular disease or risk factors was recently published 
(@) Functional Outcomes in Cardiovascular Patients Undergoing Surgical Hip Fracture Repair, FOCUS). The results were similar to the TRICC trial in that there was no difference in mortality or functional outcomes between the patients who were transfused following a restrictive strategy $(\mathrm{Hb}<8.0 \mathrm{~g} / \mathrm{dL}$ with symptoms of anemia) or the liberal strategy (transfused to keep $\mathrm{Hb}>10.0 \mathrm{~g} / \mathrm{dL}$ ) [7, 8]. There is growing interest to reduce the number of blood transfusions, as blood administration is not benign and evidence-based findings demonstrate that patients who undergo restrictive transfusion guidelines are not clinically compromised $[5,6$, 8]. The surgical literature has demonstrated a relationship between blood transfusions and increased risks of surgical site infection, morbidity, and even mortality, although a causal mechanism between receipt of blood products and these outcomes has not been established [2, 12, 13, 15, 22]. Additionally, there are associated costs with administering and storing blood [19].

Given the FOCUS study data, we have an evidencebased benchmark against which we can evaluate transfusion practice in hip fracture patients with cardiovascular disease or disease risk factors. However, there are currently no standards within orthopedic surgery for transfusing a postoperative total joint arthroplasty (TJA) patient, and it would be prudent to conduct an analysis to establish a benchmark for best practice for transfusing RBCs in TJA patients with the goal of reducing practice variability. However, in order to begin effecting a change in practice using benchmark data in total knee arthroplasty (TKA) and total hip arthroplasty (THA), it is necessary to understand the current RBC transfusion practice among surgeons who perform TJAs.

Thus, the goals of this study were to answer the following questions: (1) what is the blood utilization rate after primary TJA for individual surgeons within a large hospital network? (2) What is the comparison of hospital charges, length of stay (LOS), and discharge locations among TJA patients who were and were not transfused?

\section{Methods and Materials}

Following approval by the Quality Improvement Review Committee at our institution, the electronic medical record database was retrospectively queried to identify all surgeons who performed 10 or more primary TKA (ICD-9 code 81.54 ) or 10 or more primary THA (ICD-9 code 81.51 ) surgeries per year between June 1, 2009 and August 31, 2010. Surgeons were excluded if they performed less than 10 primary TKAs or THAs a year. Thus, there were a total of 50 surgeons who performed at least 10 TKAs during the study period, and 33 surgeons who performed at least 10 THAs. The number of cases was determined by the number of discharged primary TJA patients. During the study period, there were a total of 3,750 TKAs and 2,070 THAs, with osteoarthritis being the most common diagnosis, followed by avascular necrosis, congenital dysplasia, and rheumatoid arthritis.

All patients were admitted to the hospital on the day of surgery and underwent primary TJA. For the TKAs, most patients underwent a medial parapatellar approach, although the lateral parapatellar approach was occasionally used for valgus knees. THAs were performed with a variety of approaches, including posterior, modified Hardinge, direct lateral, and anterior, depending on the comfort of the attending surgeon. Postoperatively, each patient underwent the standard protocol of each attending surgeon, which included pain control (with or without nerve blocks) and physical therapy. The decision to transfuse patients often resulted from a discussion between anesthesiologists, internal medicine physicians, and orthopedic surgeons. Anesthesiologists played a role in determining blood transfusion within the operating room and the postoperative anesthesia care unit, while internal medicine physicians and orthopedic surgeons play a greater role preoperatively and postoperatively. Typically, the orthopedic surgeon was involved in the final decision to transfuse. For this study, patients were followed until the date of discharge from the hospital, so there was no follow-up routine to monitor.

The following outcomes were examined in this study. The blood utilization rate was determined for each surgeon who met the inclusion criteria and reflected the proportion of their patients who received at least one allogenic RBC unit in the postoperative period. Other transfusion parameters that were also calculated included the average number of postoperative allogenic RBC units transfused to each patient and the proportion of patients that received autologous blood. The rates of transfusion of other blood products were not evaluated due to the low number of platelets and plasma that these patients received. Hospital LOS was calculated by subtracting the patient's day of discharge from his/her day of admission. Hospital charges, in US dollars, were based on the hospital bill sent to patients and were not corrected for inflation. Finally, discharge locations were recorded, differentiating between discharges to either home or to a recovery facility (e.g., skilled nursing facilities and rehabilitation facilities).

For the statistical analysis of this study, the normality of the data was assessed by the Kolmogorov-Smirnov test, and continuous variables demonstrated non-normal curves; thus, nonparametric testing was implemented. Regression analysis was conducted comparing the number of TJAs performed for each surgeon to the surgeon's transfusion rate and the number of RBC units administered to each transfused patient. A statistical analysis for continuous variables (hospital charges and LOS) was analyzed using Mann-Whitney $U$ tests. Discharge location analysis was performed by chisquared tests. Statistical significance was defined as a $p$ value $<0.05$. All statistical analyses were performed using Predictive Analytics Software (PASW) version 18.0 (SPSS, Chicago, IL, USA).

\section{Results}

Of the primary TKA patients, $19.3 \%(723 / 3,750)$ received a transfusion and these patients received an average of $1.65 \pm$ $0.03 \mathrm{U}$ of RBCs in the postoperative period (Fig. 1a), while THA patients had a greater number of transfusions $(38.5 \%$, 


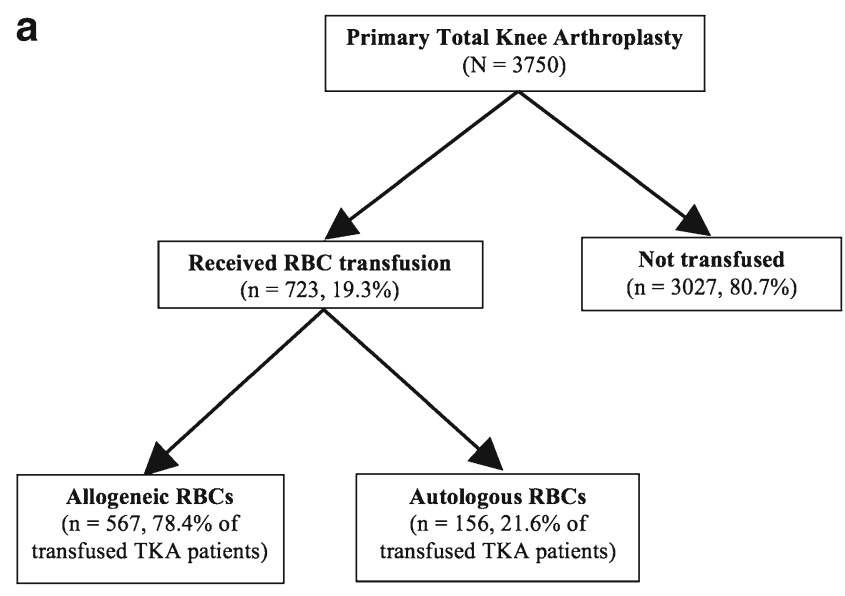

b

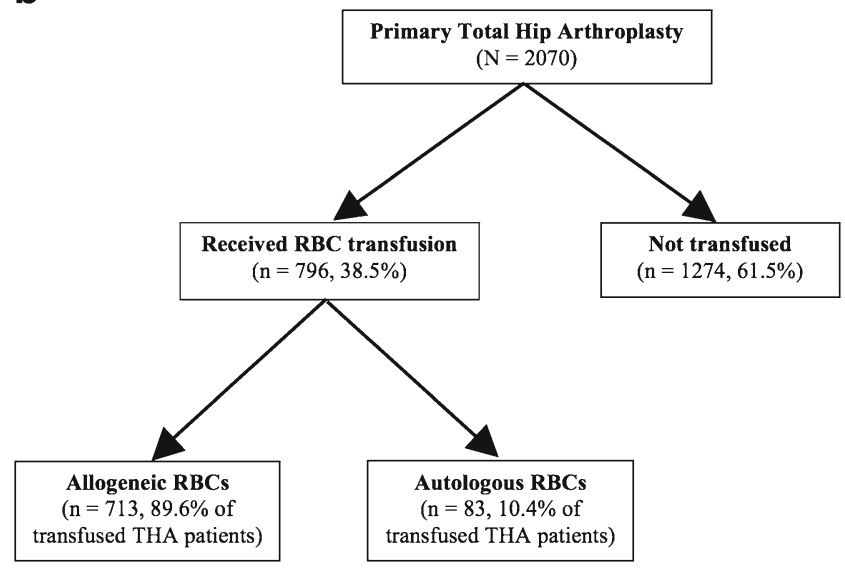

Fig. 1. Flow chart describing the transfusion populations. a Total knee arthroplasty (TKA), b total hip arthroplasty (THA).

$796 / 2,070)$ and the transfused patients received an average of $1.97 \pm 0.14 \mathrm{U}$ postoperatively (Fig. 1b). Within our health care system, allogenic RBC transfusion rates following primary TKA varied greatly between individual surgeons (Fig. 2). The lowest transfusion rate was $4.8 \%$ for a surgeon who performed 104 primary TKAs per year (Fig. 2a); this surgeon transfused an average of $1.2 \mathrm{U}$ per transfusion event (i.e., a transfused patient). On the other end of the spectrum, another surgeon performed 69 TKAs during the study period, transfused an average of $1.9 \mathrm{U}$ per transfusion event, and had a $63.8 \%$ transfusion rate. For primary THAs, the transfusion rates also varied widely between surgeons. The surgeon with the lowest transfusion rate (4.3\%) transfused an average of 1.5 $\mathrm{U}$ per transfusion event and performed 350 THAs during the study period. The surgeon with the highest transfusion rate $(86.8 \%)$ transfused an average of $2.2 \mathrm{U}$ per transfusion event and performed 386 THAs (Fig. 2b). There was no correlation between the number of cases performed and the transfusion rate (TKA: $R^{2}=0.006, p=0.613$, THA: $R^{2}=0.019, p=0.449$ ) or the average number of units transfused (TKA: $R^{2}=0.038$, $p=0.188$, THA: $R^{2}=0.002, p=0.790$ ).

Hospital LOS significantly differed between those patients that did and did not receive allogenic RBC transfusions. The average LOS was significantly longer for TKA patients who did receive a transfusion (4.0 days \pm 2.1 , median \pm standard deviation) vs. those who did not receive a transfusion (3.0 days $\pm 1.3, p<0.001$ ). The average LOS for primary THA patients who received an RBC transfusion was slightly but significantly longer ( $3.5 \pm 0.2$ days) compared to those who did not receive a transfusion $(3.2 \pm 0.2$ days, $p=0.013)$.

Hospital charges were also significantly higher for patients who received allogenic RBC transfusions compared to those that did not receive a transfusion. The average hospital charge for patients who received blood transfusions after TKA $(\$ 61,548.25 \pm \$ 33,517.45)$ was significantly higher than those that did not receive blood transfusions $(\$ 51,768.58 \pm \$ 25,921.78, p<0.001)$. The average hospital charge for THA patients who received RBC transfusions were also significantly higher $(\$ 82,588.60 \pm \$ 4,968.29)$ than the charges for patients who did not receive transfusions $(\$ 64,845.60 \pm \$ 2,384.73, p=0.002)$.

Finally, patients who received allogenic transfusions were more likely to be discharged to a recovery facility as opposed to home (Fig. 3). TKA patients who were transfused were more likely to be discharged to a facility $(37.7 \%)$ than TKA patients who were not transfused $(24.6 \%, p<$ 0.001 , Fig. 3a). The results were similar for primary THA patients (Fig. 3b). Of the transfused THA patients, $31.7 \%$ were sent to a recovery facility and $68.3 \%$ were sent home. Of the primary THA patients that were not transfused, $21.8 \%$ were sent to a facility, while $78.2 \%$ were sent home. Patients who received transfusions were more likely to be discharged to a facility as opposed to home $(p<0.001)$.

\section{Discussion}

Establishing a postorthopedic surgery RBC transfusion threshold in TJA patients has been a challenge due to the lack of evidence. The data from the FOCUS trial demonstrated that a restrictive RBC transfusion strategy did not lead to worse mortality and morbidity outcomes compared to a liberal transfusion strategy in hip fracture patients. To establish an evidence-based benchmark for postoperative blood transfusion in TJA patients, current practices must be examined. Previous studies in Europe have demonstrated that the perioperative RBC transfusion practices of surgeons who perform TJAs at various hospitals varied greatly in terms of the frequency with which patients were transfused and the number of RBCs that individual patients received $[18,21]$. In order to change RBC transfusion practice following TJAs, the extent of the variability of current transfusion practice among TJA surgeons needed to be established. Thus, we examined blood utilization rates for individual surgeons who performed primary TJA and compared hospital charges, LOS, and discharge locations for patients that were and were not transfused in our health care system.

There are several limitations with this study. This was a retrospective study, and we could not collect specific demographic data on the patients in this study. This study did not include analysis of demographic variables, including age, ASA, preoperative functioning level, preoperative $\mathrm{Hb}$, and length of time in hospital awaiting rehabilitation placement. 
a

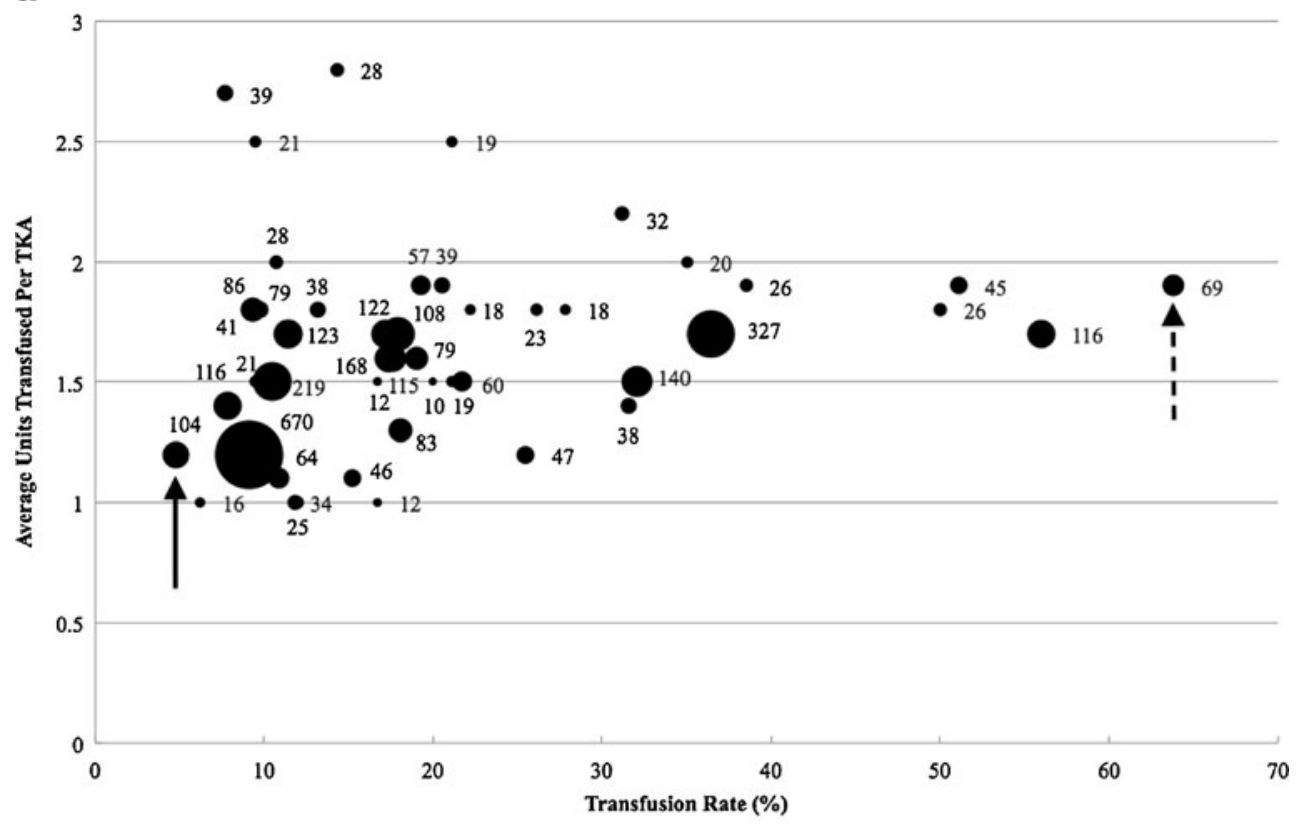

b

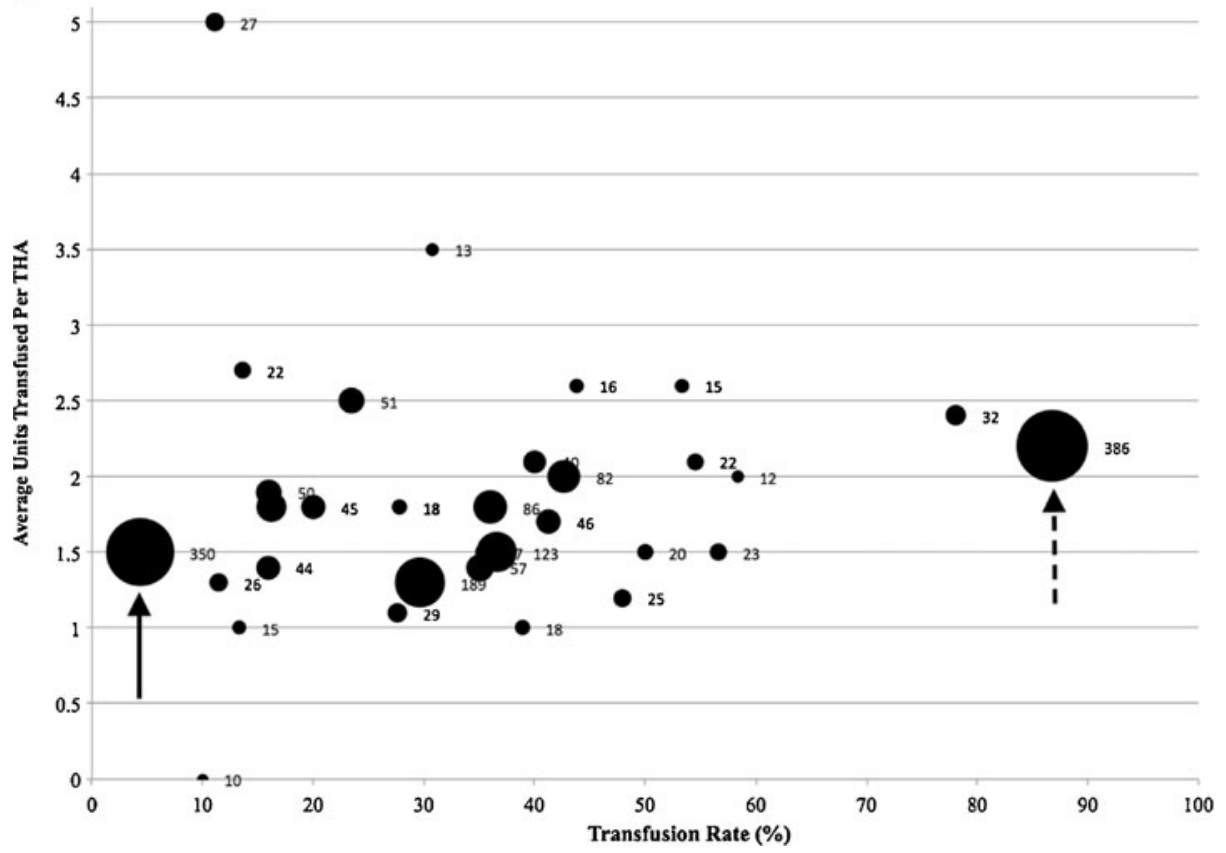

Fig. 2. Comparison of individual surgeons by the relation of the number of cases performed during the study period, the transfusion rate, and the average number of units of red blood cells (RBCs) transfused per transfusion episode. The size of the circle is indicative of the number of cases performed per surgeon. The surgeon with the highest transfusion rate is indicated with a dashed arrow and the surgeon with the lowest transfusion rate is indicated with a solid arrow. a Total knee arthroplasty (TKA), b total hip arthroplasty (THA).

Thus, we cannot exclude the possibility that the patients who received allogenic RBC transfusions might have been sicker or more anemic than those who did not require a transfusion; this possibility would confound the correlation between receipt of a transfusion and LOS, total hospital costs, and potentially, the location to which patients were discharged. A multivariate analysis is needed to analyze these individual variables to determine if blood transfusions are an independent risk factor for increased LOS, increased hospital costs, and discharge to a rehabilitation facility. The lack of individual patient demographic information also prevented an analysis of why the patients were transfused and at what $\mathrm{Hb}$ concentration they were transfused. Furthermore, other factors, such as postoperative mortality, postoperative morbidity, and return to hospital, were not evaluated in this study. This study could not evaluate whether surgeons who had lower transfusion rates had higher morbidity or mortality, or if patients of these surgeons were more likely to return to the hospital for transfusions. 


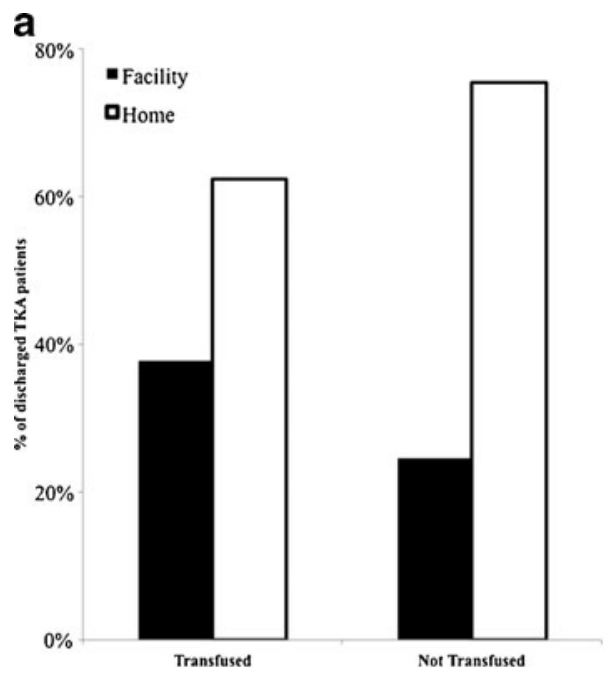

b

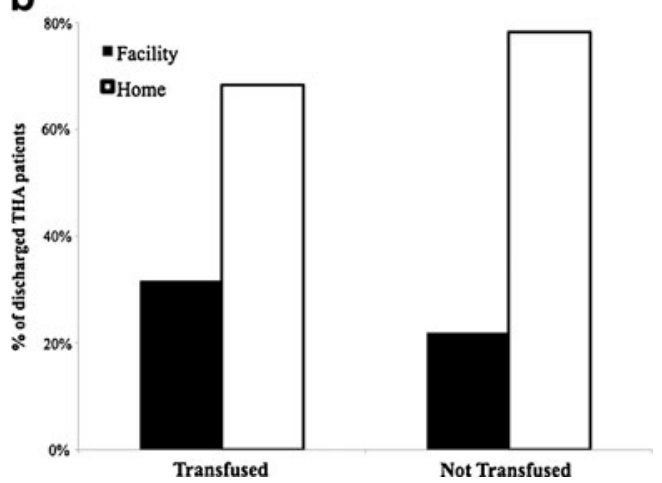

Fig. 3. Discharge location. Primary a total knee arthroplasty (TKA) and $\mathbf{b}$ total hip arthroplasty (THA) patients who were not transfused were more likely to be discharged to home as opposed to a facility (skilled nursing facility, rehabilitation facility, etc.).

Additionally, inconsistent RBC transfusion practices by individual surgeons (i.e., transfusing patients at variable $\mathrm{Hb}$ thresholds or for variable indications) would also confound this analysis. Finally, we could not collect data to determine if any blood conservation techniques were employed (e.g., regional hypotensive anesthesia, hemodilution, pharmacologic, intraoperative blood salvage methods, and autotransfusion drains). Utilizing these techniques could potentially confound postoperative transfusion rates, as patients may not have required postoperative RBCs transfusions if intra- and postoperative blood conserving techniques were used.

This study demonstrated that the transfusion rates were highly variable between surgeons who perform primary TJA in a similar geographic population of patients. There was a large spread between the surgeon who transfused the greatest number of patients and the surgeon who transfused the fewest; for TKA, the difference in transfusion rate was $59.0 \%$ (range 4.8-63.8\%), and for THA, the difference in transfusion rate was $82.5 \%$ (range $4.3-86.8 \%$ ). The variability seen in blood utilization in this study may be related to differences in technique between individual surgeons and the evidence base upon which postoperative transfusion decisions were made. For THAs, the surgeons with the highest and lowest transfusion rates both performed greater than 300 THAs yearly. The two surgeons differed with the surgical approach performed, where the surgeon with a higher transfusion rate performs minimally invasive two-incision approach, and the surgeon with the lower transfusion performs a minimally invasive posterior approach. One surgeon's minimally invasive protocol transfused RBC in patients to treat postoperative dizziness and to enable quicker recovery. The other surgeon's minimally invasive protocol limited RBC transfusions. In a multicenter study conducted in Canada that evaluated surgeons' triggers for transfusion after $\mathrm{TJA}$, it was determined that $\mathrm{Hb}$ concentration was the predominant determination of transfusion [21]. However, this study and other studies conducted in Europe and Canada, looked at the aggregate of surgeons and not individual surgeons $[9,11$, $16,18]$. In order to implement evidence-based transfusion thresholds, it is necessary to understand the practices and beliefs that underlie each surgeon's decision to transfuse or not to transfuse. Thus, this study uniquely looked at individual surgeons and their transfusion rates in relation to the number of TJAs they performed annually.

This study also demonstrated that primary TJA patients who received transfusions had shorter LOS, lower hospital charges, and were more likely to be discharged home. These findings have also been shown in cardiac surgery, vascular surgery, and ICU patients $[3,4,10,14,17]$ but have never been done in TJA patients. With the creation of accountable care organizations or a type of payment model that ties provider reimbursements to certain quality measures and reductions in costs for specific patient populations, standardizing medical practice will become necessary in order to achieve potential cost savings. One way of reducing the costs associated with transfusions may be to encourage the preoperative optimization of the patient's $\mathrm{Hb}$ concentration by the surgeons themselves or through referral to a primary care doctor or to a clinic designed to diagnose and treat preoperative anemia.

Since previous studies have shown that a restrictive RBC transfusion threshold does not increase morbidity or mortality in hip surgery patients, it is imperative that benchmarks also be established in TJA patients and that practice guidelines are made to reflect the current evidence base. To that end, the current standard of practice for individual surgeons performing primary TJA in a large hospital network was assessed for their blood transfusion rates in the postoperative period; our study demonstrated that there was great variation in transfusion practice among these surgeons, which might have been responsible for the variability in the outcomes demonstrated in this study.

Our study also demonstrated that transfused patients tend to stay in the hospital longer, thereby amassing greater costs and were less likely to have been discharged home. Thus, establishing evidence-based criteria to reduce blood transfusions after TJA may minimize costs and decrease hospital LOS in TJA patients. This study was designed to capture the practice of orthopedic surgeons and their blood utilization rates to better understand their views. Thus, the results of our quality improvement study have been presented as a benchmarking tool to vice presidents of operations at individual hospitals within our system, surgeons who were studied, and staff to educate them on the efficacy of a limited transfusion protocol. By showing individual surgeons their transfusion rates, surgeons have responded by changing their 
transfusion practices [20], with a few outliers. Future quality improvements studies will examine if awareness of this benchmarking study can enact standardized practice guideline changes in surgeon transfusion practices by reducing allogenic RBC transfusions from outlier surgeons.

Appropriately allocating blood product resources for transfusions after primary TKA may require the standardization of formal transfusion criteria. Future directions for studies include prospectively examining transfusion triggers for individual surgeons performing primary TJA and determining universal guidelines by which orthopedic surgeons should transfuse primary TJA patients to ensure optimal patient outcomes.

\section{Disclosures}

Conflict of Interest: Antonia F. Chen, MD, MBA has received research grant funding from OREF; The Pittsburgh Foundation and Scoliosis Research Society and receives royalties from Slack publishing, outside the work.

Brian A. Klatt, MD is a board member for Regional Arthritis Board; provided expert testimony for Reminger Attorneys at Law; has received research grant funding from OREF; The Pittsburgh Foundation and Depuy and will receive royalties from Slack publishing, outside the work. Mark H. Yazer, MD declared that he has no conflict of interest.

Jonathan H. Waters, MD is a consultant for Octoplasa and Hemocue; employee of University of Pittsburgh; has received research grant funding from Centers for Disease Control (CDC), Sorin, Haemonetics, Masimo, AMAG, has received payment for lectures from Procirca, Hemocue, Sorin and travel support and other expenses from West Virginia Univ./ California Blood Bank Society, outside the work.

Human/Animal Rights: All procedures followed were in accordance with the ethical standards of the responsible committee on human experimentation (institutional and national) and with the Helsinki Declaration of 1975, as revised in 2008 (5).

Informed Consent: Informed consent was obtained from all patients for being included in the study.

Required Author Forms Disclosure forms provided by the authors are available with the online version of this article.

Open Access This article is distributed under the terms of the Creative Commons Attribution License which permits any use, distribution, and reproduction in any medium, provided the original author(s) and the source are credited.

\section{References}

1. Adams R, Lundy J. Anesthesia in cases of poor surgical risk: some suggestions for decreasing the risk. Surg Gynecol Obstet. 1942;64:1011-1019.

2. Bernard AC, Davenport DL, Chang PK, Vaughan TB, Zwischenberger JB. Intraoperative transfusion of $1 \mathrm{U}$ to $2 \mathrm{U}$ packed red blood cells is associated with increased 30-day mortality, surgicalsite infection, pneumonia, and sepsis in general surgery patients. J Am Coll Surg. 2009;208:931-937, 937 e931-932; discussion 938-939.

3. Bracey AW, Radovancevic R, Riggs SA, Houston S, Cozart H, Vaughn WK, Radovancevic B, McAllister HA, Jr., Cooley DA. Lowering the hemoglobin threshold for transfusion in coronary artery bypass procedures: effect on patient outcome. Transfusion. 1999;39:1070-1077.
4. Bush RL, Pevec WC, Holcroft JW. A prospective, randomized trial limiting perioperative red blood cell transfusions in vascular patients. American journal of surgery. 1997;174:143-148.

5. Carless PA, Henry DA, Carson JL, Hebert PP, McClelland B, Ker K. Transfusion thresholds and other strategies for guiding allogeneic red blood cell transfusion. Cochrane Database Syst Rev. 2010:CD002042.

6. Carson JL, Carless PA, Hebert PC. Transfusion thresholds and other strategies for guiding allogeneic red blood cell transfusion. Cochrane Database Syst Rev. 2012;4:CD002042.

7. Carson JL, Terrin ML, Magaziner J, Chaitman BR, Apple FS, Heck DA, Sanders D. Transfusion trigger trial for Functional Outcomes in Cardiovascular Patients Undergoing Surgical Hip Fracture Repair (FOCUS). Transfusion. 2006;46:2192-2206.

8. Carson JL, Terrin ML, Noveck H, Sanders DW, Chaitman BR, Rhoads GG, Nemo G, Dragert K, Beaupre L, Hildebrand K, Macaulay W, Lewis C, Cook DR, Dobbin G, Zakriya KJ, Apple FS, Horney RA, Magaziner J. Liberal or restrictive transfusion in high-risk patients after hip surgery. N Engl J Med. 2011;365:2453-2462.

9. Eindhoven GB, Diercks RL, Richardson FJ, van Raaij JJ, Hagenaars JA, van Horn JR, de Wolf JT. Adjusted transfusion triggers improve transfusion practice in orthopaedic surgery. Transfus Med. 2005;15:13-18.

10. Hebert PC, Wells G, Blajchman MA, Marshall J, Martin C, Pagliarello G, Tweeddale M, Schweitzer I, Yetisir E. A multicenter, randomized, controlled clinical trial of transfusion requirements in critical care. Transfusion Requirements in Critical Care Investigators, Canadian Critical Care Trials Group. N Engl J Med. 1999;340:409-417.

11. Helm AT, Karski MT, Parsons SJ, Sampath JS, Bale RS. A strategy for reducing blood-transfusion requirements in elective orthopaedic surgery. Audit of an algorithm for arthroplasty of the lower limb. J Bone Joint Surg Br. 2003;85:484-489.

12. Hill GE, Frawley WH, Griffith KE, Forestner JE, Minei JP. Allogeneic blood transfusion increases the risk of postoperative bacterial infection: a meta-analysis. J Trauma. 2003;54:908-914.

13. Innerhofer P, Klingler A, Klimmer C, Fries D, Nussbaumer W. Risk for postoperative infection after transfusion of white blood cell-filtered allogeneic or autologous blood components in orthopedic patients undergoing primary arthroplasty. Transfusion. 2005;45:103-110.

14. Johnson RG, Thurer RL, Kruskall MS, Sirois C, Gervino EV, Critchlow J, Weintraub RM. Comparison of two transfusion strategies after elective operations for myocardial revascularization. The Journal of thoracic and cardiovascular surgery. 1992;104:307-314.

15. Koch CG, Li L, Duncan AI, Mihaljevic T, Loop FD, Starr NJ, Blackstone EH. Transfusion in coronary artery bypass grafting is associated with reduced long-term survival. Ann Thorac Surg. 2006;81:1650-1657.

16. Ma M, Eckert K, Ralley F, Chin-Yee I. A retrospective study evaluating single-unit red blood cell transfusions in reducing allogeneic blood exposure. Transfus Med. 2005;15:307-312.

17. Pattakos G, Koch CG, Brizzio ME, Batizy LH, Sabik JF, Blackstone $\mathrm{EH}$, Lauer MS. Outcome of patients who refuse transfusion after cardiac surgery: a natural experiment with severe blood conservation. Archives of internal medicine. 2012;172:1154-1160.

18. Rosencher N, Kerkkamp HE, Macheras G, Munuera LM, Menichella G, Barton DM, Cremers S, Abraham IL. Orthopedic Surgery Transfusion Hemoglobin European Overview (OSTHEO) study: blood management in elective knee and hip arthroplasty in Europe. Transfusion. 2003;43:459-469.

19. Shander A, Hofmann A, Gombotz H, Theusinger OM, Spahn DR. Estimating the cost of blood: past, present, and future directions. Best Pract Res Clin Anaesthesiol. 2007;21:271-289.

20. Taylor G, Buchanan-Chell M, Kirkland T, McKenzie M, Sutherland B, Wiens R. Reduction in surgical wound infection rates associated with reporting data to surgeons. The Canadian journal of infectious diseases $=$ Journal canadien des maladies infectieuses. 1994;5:263-267.

21. Vuille-Lessard E, Boudreault D, Girard F, Ruel M, Chagnon M, Hardy JF. Red blood cell transfusion practice in elective orthopedic surgery: a multicenter cohort study. Transfusion. 2010;50:2117-2124.

22. Weber WP, Zwahlen M, Reck S, Misteli H, Rosenthal R, Buser AS, Kaufmann M, Oertli D, Widmer AF, Marti WR. The association of preoperative anemia and perioperative allogeneic blood transfusion with the risk of surgical site infection. Transfusion. 2009;49:1964-1970. 